documented in animal models with histology, but rarely with non-invasive means like magnetic resonance imaging (MRI). A few human MRI studies have investigated changes in long WM tracts after ICH, like the corticospinal tract, but have not attempted to obtain an unbiased quantification of WM changes within and around the hematoma over time. This study attempts such quantification from 3 to 30 days post ictus.

Methods Thirteen patients with ICH underwent diffusion tensor imaging (DTI) MRI at 3, 14, and 30 days. Fractional anisotropy (FA) maps were used to calculate volume of tissue with $\mathrm{FA}>0.5$ both within hematoma (lesion) and in the perilesional tissue.

Results At day 3, both the percent of lesional and peri-lesional tissue with an FA>0.5 was significantly less than contralateral tissue. This peri-lesional contralateral difference persisted at day 14 , but there was no significant difference at day 30 . The loss of peri-lesional tissue with $\mathrm{FA}>0.5$ increased with increasing hematoma size at day 3 and day 14. All patients had some tissue within lesion with $\mathrm{FA}>0.5$ at all time points. This did not decrease with duration after ictus, suggesting the persistence of white matter within the hematoma/lesion.

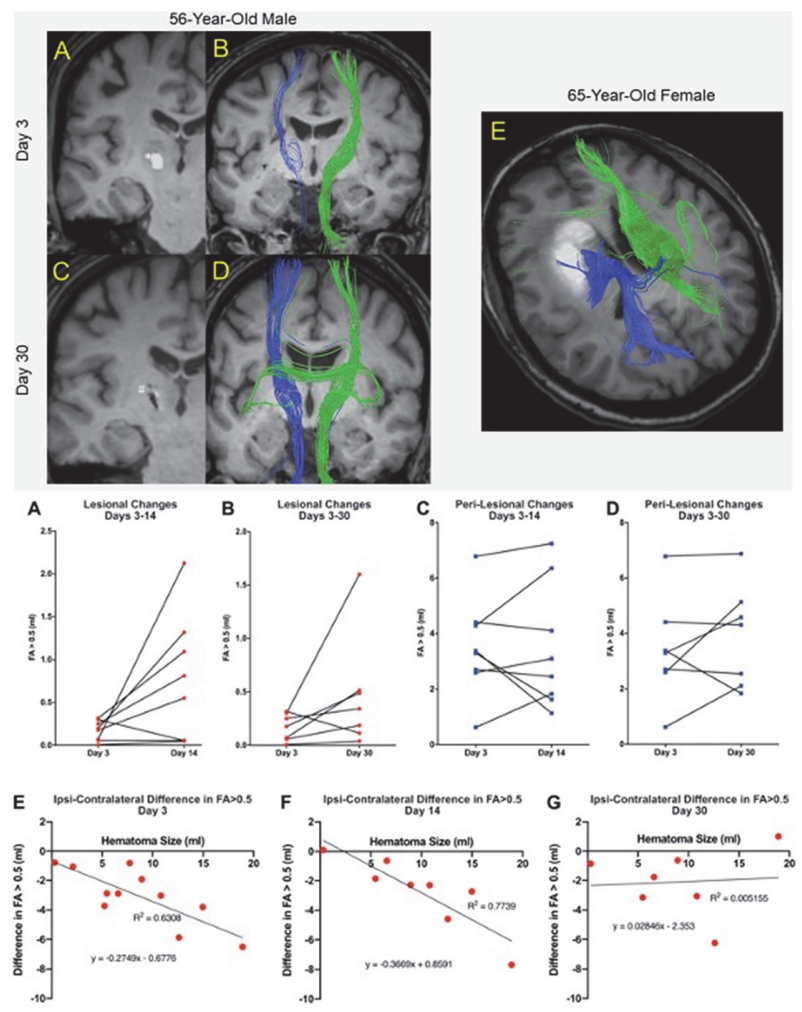

Abstract 0-022 Figure 1

Conclusion These results outline an approach to quantify WM injury, both within and surrounding the hematoma, after ICH using DTI MRI. This may be important for monitoring treatment strategies, such as hematoma evacuation, and assessing efficacy non-invasively.

Disclosures N. Novakovic: None. J. Linzey: None. T. Chenevert: None. J. Gemmete: None. G. Xi: None. R. Keep: None. A. Pandey: None. N. Chaudhary: None.

\section{0-023 ARTERIAL SPIN LABELING (ASL) MRI ASSISTS IN IDENTIFICATION OF CEREBRAL MICRO-ARTERIOVENOUS MALFORMATIONS}

V Mayercik*, R Taiwo, M Marks, R Dodd, J Heit, H Do, N Telischak. Stanford University, Stanford, $C A$

\subsection{6/neurintsurg-2021-SNIS.23}

Introduction Micro-arteriovenous malformations (microAVMs) are a subtype of cerebral AVM characterized by an arterial nidus less than $1 \mathrm{~cm}$ in diameter. Due to their small size, these lesions may be difficult to identify on conventional MRI. They can also be missed or occult on cerebral angiography. Arterial spin labeling (ASL) is an MRI sequence which acts as a surrogate marker for arterial perfusion and which may be useful in the identification of small arterial lesions such as microAVMs.

Materials and Methods We conducted a retrospective cohort study of 34 microAVMs in 19 patients, diagnosed at a single academic medical center from 1996 to 2020. Inclusion criteria were presence of microAVM confirmed by either cerebral angiogram or surgery. Patients without ASL-MRI were excluded. An electronic medical record was queried for patient demographics, diagnostic work-up, results of ASL-MRI, and surgical pathology when available. Two experienced neuroradiologists counted the number and location of lesions seen on ASL-MRI in a blinded fashion. Cohen's kappa was calculated for inter-rater reliability.

Results 22 patients with microAVMs were initially selected. 3 patients were excluded due to lack of ASL imaging. 12 males and 7 females were included with a total of 34 microAVMs. Mean patient age was 39.8 years ( $\mathrm{SD} \pm 19.0$ years). Among 34 microAVMs, 27 lesions had positive findings on ASL (79.4\%). Inter-rater agreement was substantial (Cohen's kappa $=0.70$ ) with raters agreeing in $88.6 \%$ of cases. 9 patients were initially diagnosed with one or more microAVMs due to the presence of positive ASL signal (47.4\%). 3 patients with ASL findings suggestive of a microAVM had angiographically occult lesions which were confirmed at the time of surgery (15.8\%).

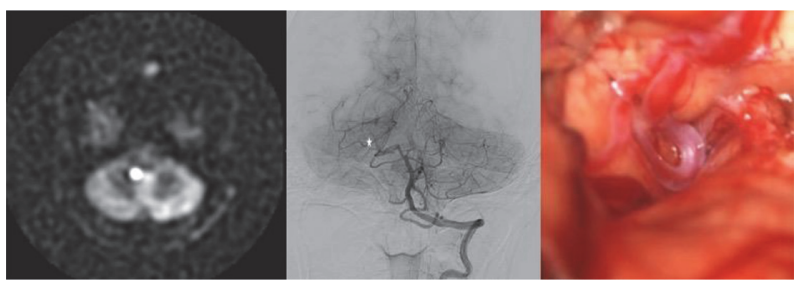

Abstract 0-023 Figure 1

Conclusion ASL assists in the identification of cerebral microAVMs. This sequence can be used to suggest an initial diagnosis of microAVM. It can also identify angiographically occult microAVMs confirmed at the time of surgical excision.

Disclosures V. Mayercik: None. R. Taiwo: None. M. Marks: None. R. Dodd: None. J. Heit: None. H. Do: None. N. Telischak: None. 